\title{
DESARROLLO FINANCIERO, ACUMULACIÓN DE CAPITAL FORÁNEO Y POBREZA: MODELO TEÓRICO RBC EN ECONOMÍA PEQUEÑA ABIERTA
}

Financial development, foreign capital accumulation and poverty: a theoretical RBC model for a small open economy

\section{Gabriel Jaime González Uribe}

Economista. Instituto Tecnológico Metropolitano. Medellín, Colombia.gabrielgonzalez@itm.edu.co

\begin{abstract}
Cómo citar / How to cite
González, G. (2015). Desarrollo financiero, acumulación de capital foráneo y pobreza: Modelo teórico RBC en economía pequeña abierta. Revista CEA, 1(1), 11-21.
\end{abstract}

Recibido: 16 de junio de 2014

Aceptado: 10 de septiembre de 2014

\section{Resumen}

La economía mundial se caracteriza por altos niveles de pobreza. Algunos autores han relacionado el desarrollo financiero, entendido como todas aquellas medidas que le permiten al sistema financiero reducir los costos de información y transacción, facilitando la movilización y locación eficiente del capital con los niveles de pobreza de los países. Este artículo utiliza el marco teórico Kollintzas y Vassilatos (2000) para hacer una aproximación desde un modelo simple de Ciclos Económicos Reales (o RBC por sus siglas en inglés), endogeneizando los costos de transacción, relacionándolos con el desarrollo financiero de los países. Los resultados indican que mejorías en el desarrollo financiero afectan las decisiones óptimas de los individuos e incide indirectamente en la remuneración de los factores de producción, entre ellos el trabajo.

Palabras clave: desarrollo financiero, modelos de ciclos económicos reales, reducción de la pobreza.

\begin{abstract}
Global economy is characterized by high levels of poverty. Some authors have linked financial development, understood as all those measures that allow the financial system to reduce information and transaction costs, and facilitate the mobilization and efficient location of the capital, to poverty levels of the countries. This paper uses the theoretical framework of Kollintzas and Vassilatos (2000) as a reference for a simple Real Business Cycle (RBC) model endogenizing transaction costs, referring to the financial development of countries. The results show that improvements in financial development affect the optimal decisions of individuals and indirectly affect the remuneration of the factors of production, including labor.
\end{abstract}

Keywords: financial development, real business cycle models, poverty reduction 


\section{INTRODUCCIÓN}

La economía mundial se caracteriza, en general, por altos niveles de pobreza. Según Beck, Demirguc-Kunt y Levine (2004), en el 2001, unos 2,7 miles de millones de personas en el mundo (más de la mitad de la población mundial en esa época) vivían con menos de \$2 dólares al día, y 1,1 miles de millones de personas vivían con menos de $\$ 1$ dólar el día. Si bien en los últimos años ha habido una mejoría de las condiciones de pobreza en el marco mundial, según un estudio del Banco Mundial (World Bank, 2013) aún en 2013 unas 1,2 miles de millones de personas vivían en extrema pobreza.

En los últimos 10 años ha aumentado el número de estudios en el tema del desarrollo financiero o grado de bancarización de los países, y su relación con la pobreza y desigualdad del ingreso.

Por ejemplo, Gill (2006) busca determinar cuál es la relación de causalidad entre alfabetismo financiero, el tipo de empleo y la confianza, en el estatus bancario de las personas de nueve provincias de Sudáfrica. El autor encuentra que el estatus bancario de la persona depende del alfabetismo financiero y del tipo de empleo, pero no de la confianza en el sistema financiero. Esto es, que entre más y mejor sea el conocimiento de la persona del sistema financiero y mejor sea su calidad de empleo, mejor será su estatus bancario.

Por su parte, Medhi, Ratan y Toyama (2009) examinan las diferencias entre los países que adoptan y usan los servicios de banca móvil (m-banking), por personas con bajos niveles educativos y bajos ingresos, y los posibles factores que las explican. Ellos concluyen que el crecimiento en el número de cuentas no está significativamente asociado con la reducción de la línea de pobreza entre los países. Es decir, es probable que no todos los desarrollos financieros estén asociados a unos menores niveles de la pobreza.

A su vez, Nguyen, Bigman, y Van Den Berg (2007) muestran a através de un modelo econométrico de panel de datos de efectos fijos, cómo las pólíticas del programa Vietman Bank for Social Policies (VBSP) han sido muy efectivas en su ánimo de combatir la pobreza, pues encuentran que la participación en este programa, en promedio, parece aumentar el ingreso $y$ gastos de los hogares en alrededor de un $30 \%$ del valor del préstamo, y un incremento en el tamaño del préstamo tiene un efecto similar. Pese a que un tercio de los préstamos los alcanzan hogares pobres, el programa reduce la incidencia de la pobreza de sus participantes en cerca de cuatro puntos porcentuales. De manera similar, el programa reduce el índice de la brecha de pobreza y el índice de severidad de la pobreza en casi $20 \%$.

Por último, Beck, Demirguc-Kun y Levine (2004) estiman el efecto de desarrollo financiero en los cambios de la distribución del ingreso y el alivio de la pobreza. Estimando un modelo econométrico de regresiones cross-country por el método de los mínimos cuadrados ordinarios, usando variables instrumentales para controlar causalidad inversa y sesgo de simultaneidad, logran establecer que un mayor desarrollo financiero induce a que el ingreso de los pobres crezca más rápido que el PIB per cápita, que la desigualdad del ingreso decrezca más rápido y que las tasas de pobreza decrezcan a una tasa mayor. Además, los resultados se mantienen aun cuando se controla por la tasa promedio de crecimiento de la economía, lo que indica que el desarrollo financiero alivia la pobreza más allá del sus efectos sobre el crecimiento agregado. Esto es, se encuentra que el desarrollo financiero reduce la pobreza al ejercer un efecto desproporcionadamente positivo en los pobres. 
El modelo de pequeña economía abierta permite observar los efectos de las políticas económicas y shocks exógenos en los equilibrios interno y externo, así como en las decisiones óptimas que toman los agentes. Es por ello que en este artículo se utilizará este modelo macroeconómico para introducir el efecto del desarrollo financiero en las principales variables macroeconómicas del país, entre ellas la pobreza.

El propósito de este artículo es utilizar el marco teórico propuesto por Kollintzas y Vassilatos (2000) partiendo de una simplificación del mismo, con el fin de endogeneizar los costos de transacción en el modelo, el cual supone el elemento diferenciador de este trabajo con aquel, suponiendo que dichos costos son una función decreciente del desarrollo financiero $y$, de este manera, observar cómo se afectan las conclusiones del modelo ante variaciones en el desarrollo financiero de los países.

\section{MARCO TEÓRICO}

Se parte del marco conceptual descrito en Kollintzas y Vassilatos (2000). En ese artículo, los autores desarrollan un modelo extendido de pequeña economía abierta en el cual vinculan los efectos de los costos de transacción del capital foráneo y los shocks permanentes y transitorios de la política fiscal en las variables macroeconómicas. Nuestro propósito es utilizar ese marco conceptual desde una perspectiva más sencilla, dejando de lado los efectos de la política fiscal, sin vincular crecimiento poblacional ni cambio técnico en el trabajo, pero permitiendo que haya costos de ajustes en el capital privado doméstico $y$, desde luego, profundizando más en las implicaciones económicas de los costos de transacción, más específicamente, estableciendo cómo el desarrollo financiero vía costos de transacción afectan las variables de decisión de los agentes en la economía.

A continuación se describe el modelo empleado en Kollintzas and Vassilatos (2000) pero bajo una versión simplificada, y también se describe la modificación que se le hace al mismo en relación a los costos de transacción.

\section{Modelo básico de ciclos económicos reales}

Un modelo básico RBC (Real Business Cycle) está compuesto por tres tipos de agentes: los hogares que maximizan sus funciones de utilidad y eligen sus cestas óptimas de consumo (y de ocio, en nuestro caso); las firmas que maximizan sus beneficios $y$ determinan los precios a los cuales se remuneran los factores (en este caso, el nivel de salario y la tasa de interés); y el gobierno, que define el nivel de recaudo y de gasto óptimo. En nuestro modelo sencillo se hará abstracción del gobierno, a fin de simplificar el análisis y centrarnos en la influencia del desarrollo financiero en el comportamiento de los hogares en la economía.

A continuación se describirán cada uno de los agentes de la economía, sus funciones a maximizar y sus elecciones óptimas; posteriormente, se describirá la solución del modelo; y finalmente, se detallarán las implicaciones de la inclusión del desarrollo financiero en nuestro modelo, haciendo una comparación con los resultados del modelo RBC básico.

\section{Los hogares}

Sea una economía de precios relativos poblada por un gran número de individuos $(\mathrm{N})$ iguales y racionales, cuyas preferencias por consumo y ocio son tales que:

$$
U=U(C, L)
$$


Donde $C$ es consumo agregado y $L$ es ocio (fracción de tiempo dedicado a actividades de ocio).

Suponga que la forma específica que toma la función de utilidad es del tipo Isoelástica o CRRA (Constan Relative Risk Aversion Function, por sus siglas en inglés) anidada a unas preferencias tipo Cobb-Douglas de modo que:

$$
U(C, L)=\frac{\left(C^{\gamma} L^{1-\gamma}\right)^{1-\sigma}-1}{1-\sigma}
$$

Donde $\gamma$ es la valoración del consumo para el individuo y $1-\gamma$ la del ocio, teniendo en cuenta que $0<\gamma<1$. Y $\sigma$ es un parámetro de elasticidad de sustitución, si $\sigma \rightarrow 1$ la función de utilidad es logarítmica y si $\sigma \rightarrow 0$ tenemos la clásica función de utilidad CobbDouglas.

\section{La firma}

Se asumirá un marco de competencia perfecta en la producción de un solo bien final homogéneo $Y_{t}$. En su producción se utilizan capital privado $K_{t}^{p}$ y trabajo $H_{t}$. La tecnología utilizada es de rendimientos constantes a escala, y se supondrá una función de producción neoclásica tipo CobbDouglas de modo que:

$$
Y_{t}=A_{t} K_{t}^{p^{\alpha}}\left(H_{t}\right)^{1-\alpha}
$$

Con $A_{t}>0, \alpha \epsilon(0,1)$.

Donde $A_{t}$ es la Productividad Total de los Factores (PTF); $H_{t}$ es la fracción de tiempo dedicada a actividades de trabajo en el período $t$, es decir,

$$
H_{t}=1-L_{t}
$$

$\alpha$ es la participación del capital privado en la producción del bien la cual se supone constante y conocida.
La evolución del capital privado la describe la siguiente ecuación:

$$
K_{t+1}^{p}=\varphi\left(\frac{I_{t}}{K_{t}^{p}}\right) K_{t}^{p}+(1-\delta) K_{t}^{p}, \operatorname{con} \delta \epsilon(0,1)
$$

Donde $I_{t}$ es la inversión privada agregada y $\varphi($.$) es una función que incorpora costos de$ ajuste internos en la inversión, que cumple que $\varphi^{\prime}()>$.0 y $\varphi^{\prime \prime}()<$.0 . Para efectos de este artículo, asumiremos como es usual que la forma específica que toma esta función es la siguiente:

$$
\varphi_{t}=1+\frac{\chi}{2}\left(\frac{I_{t}}{K_{t}^{p}}\right)^{2}
$$

Donde $I_{t}$ es la inversión privada e igual a:

$$
I_{t}=K_{t+1}^{p}-K_{t}^{p}
$$

$\chi$ es un parámetro de ajuste positivo, si $\chi=0$ no habrá costos de ajuste del capital.

\section{Comercio internacional}

Aquí es donde los autores se diferencian con otros trabajos como los de Mendoza (1991), Bruno y Portier (1995) y Cardia (1991). En primer lugar, Mendoza (1991) formaliza la hipótesis de pequeña economía abierta endogenizando la tasa de tiempo de las preferencias; por su parte, Bruno y Portier (1995) asumen que los activos foráneos entran negativamente en la función de utilidad; mientras que Cardia (1991) sigue el enfoque de tiempo de vida finito de Blanchard (1985) y Yaari (1965). En este caso, los autores cierran el modelo introduciendo costos de transacción en el sector foráneo (Kollintzas and Vassilatos, 2000, p. 1519). Por lo que la tenencia de activos foráneos de los hogares se describe a partir de la siguiente ecuación:

$$
P_{t}^{f} \psi\left(K_{t}^{f}, K_{t+1}^{f}\right) K_{t+1}^{f}=K_{t}^{f}+X_{t}+T_{t}^{f}
$$


Donde,

$$
P_{t}^{f}=\frac{1}{1+r_{t}^{*}}
$$

Y la condición de transversalidad implica que:

$$
\lim _{t \rightarrow \infty} E_{0}\left(\prod_{u=0}^{t} P_{u}^{f}\right) K_{t+1}^{f}=0
$$

Donde $K_{t}^{f}$ son las tenencias netas agregadas de activos foráneos en el periodo $t ; X_{t}$ son las exportaciones netas en el periodo $t ; T_{t}^{f}$ son las transferencias agregadas extranjeras en el periodo $t ; r_{t}^{*}$ es la tasa de retorno internacional, e igual a $r_{t}^{*}=1 / P_{t}^{f}-1$. Lo que indica que $P_{t}^{f}$ es el precio de un activo que entrega una unidad de consumo en el siguiente período (t+1) (Kollintzas and Vassilatos, 2000 página 1520).

Los costos de transacción en este modelo se conciben con la inclusión de la función $\psi($.$) ,$ los cuales afectan el precio de los activos foráneos. Se asume que es una función positiva o creciente con el nivel de transacciones. Kollintzas and Vassilatos (2000) consideran la siguiente forma para la función $\psi($.$) :$

$$
\psi\left(K_{t}^{f}, K_{t+1}^{f}\right)=1+\frac{\psi}{2}\left(\frac{K_{t+1}^{f}-K_{t}^{f}}{K_{t}^{f}}\right)^{2}
$$

Donde consideran que $\psi$ es una constante positiva, en adelante la nombraremos $\psi^{*}$.

Note que en el estado estacionario no hay costos de transacción, ya en el estado estacionario lo que está dentro del paréntesis es cero.

Es aquí donde se modificará el modelo. Pues se supondrá que $\psi^{*}$ no es ahora una constante sino que es una variable que depende del grado de desarrollo financiero del país $\left(F D_{t}\right)$ en el momento $t$, de modo que:

$$
\psi^{*}=\psi^{*}\left(F D_{t}\right)
$$

$$
\begin{gathered}
\psi^{*^{\prime}}\left(F D_{t}\right)<0 \\
\psi^{* \prime \prime}\left(F D_{t}\right)>0
\end{gathered}
$$

Donde el desarrollo financiero $F D_{t}$ se entenderá en el sentido amplio según Beck, Demirguc-Kun y Levine (2004). Estos autores sugieren que el desarrollo financiero son todas aquellas medidas que le permiten al sistema financiero reducir los costos de información y transacción, y que facilitan la movilización y locación eficiente del capital.

En realidad, es de esperarse que el grado de desarrollo financiero afecte los costos de transacción en otro momento, es decir, que el efecto no sea inmediato sino que de alguna manera exista algún tipo de rezago que describa la dificultad con que la economía asimila el cambio o desarrollo financiero y la interiorice. Sin embargo, para simplificar el análisis se supondrá que el efecto del desarrollo financiero sobre los costos de transacción de los activos foráneos es instantáneo.

La ecuación (12) indica que los costos de transacción son una función decreciente del desarrollo financiero, pero que con cada innovación o desarrollo es más difícil lograr reducir los costos de transacción, es por ello que la segunda derivada es positiva. Gráficamente tenemos lo siguiente:

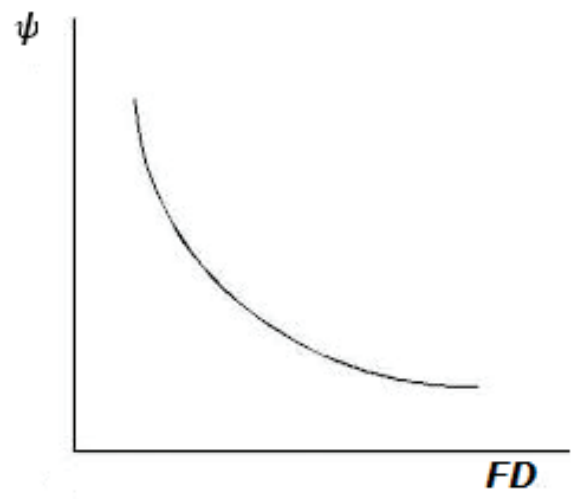

Figura 1. Relación entre el Desarrollo Financiero (FD) y los costos de transacción.

Figure 1. Relationship between Financial Development (FD) and transaction costs.

Fuente: Elaboración propia 
Se podría suponer que existe un valor idiosincrático de desarrollo financiero, de tal manera que exista heterogeneidad en el grado de este, entre el país local y el resto del mundo (en ese caso la función tomaría una forma similar a $\psi^{*}=\bar{\psi}+\psi^{*}\left(F D_{t}^{i}\right)$, donde $\bar{\psi}$ es un costo de transacción fijo para todos los países, y $\psi^{*}\left(F D_{t}^{i}\right)$ es una función del grado de desarrollo financiero del país $i$ en el período $t$ ). Sin embargo, para facilitar el análisis solo se supondrá variaciones en $t$ y no en $i$.

De manera análoga a la forma como se modelan los costos de ajuste, se supondrá que la forma específica de la función de costos de transacción $\psi^{*}$ es la siguiente:

$$
\psi^{*}=\bar{\psi}+\frac{1}{F D_{t}}
$$

Esta forma funcional permite modelar de manera adecuada la incidencia del desarrollo financiero sobre los costos de transacción en nuestro modelo, pues permite conservar las propiedades deseadas que se describen en la ecuación (11) y en la Figura 1 (ver A.1 APÉNDICE 1).

Por último, las transferencias foráneas se generan de acuerdo con:

$$
T_{t}^{f}=S_{t}^{f} Y_{t}
$$

Donde $S_{t}^{f}$ se forma estocásticamente así como $A_{t}, P_{t}^{f}$ y $F D_{t}$ de acuerdo con la siguiente regla:

$$
\begin{array}{r}
\ln \left(A_{t+1}\right)=\left(1-\rho_{a}\right) \ln (A)+\rho_{a} \ln \left(A_{t}\right)+\varepsilon_{t+1}^{a} \\
\ln \left(S_{t+1}^{f}\right)=\left(1-\rho_{f}\right) \ln \left(S^{f}\right)+\rho_{f} \ln \left(S_{t}^{f}\right)+\varepsilon_{t+1}^{f} \\
\ln \left(P_{t+1}^{f}\right)=\left(1-\rho_{p}\right) \ln \left(P^{f}\right)+\rho_{p} \ln \left(P_{t}^{f}\right)+\varepsilon_{t+1}^{p} \\
\ln \left(F D_{t+1}\right)=\left(1-\rho_{d}\right) \ln \left(F D^{d}\right)+\rho_{d} \ln \left(F D_{t}^{d}\right)+\varepsilon_{t+1}^{d}
\end{array}
$$

Donde $\varepsilon_{t+1}^{a}, \varepsilon_{t+1}^{p}, \quad \varepsilon_{t+1}^{f} \mathrm{y} \varepsilon_{t+1}^{d} \quad$ son shocks estocásticos en las series $A_{t+1}, P_{t+1}^{f}, S_{t+1}^{f} \mathrm{y}$ $F D_{t}$ respectivamente, y $A, P^{f}, S^{f}$ y $F D^{d}$ son las medias de los procesos estocásticos respectivamente.

En esto también se diferencia este artículo con el de Kollintzas and Vassilatos (2000), pues estos autores al considerar exógenos los costos de transacción no generan un proceso estocástico de los mismos, en este caso, dicho proceso está representado por la ecuación (18), la cual genera un proceso estocástico para el desarrollo financiero; y por las ecuaciones (11) y (13) dicho proceso se extiende a los costos de transacción.

\section{El equilibrio competitivo}

\section{$>$ La firma}

El objetivo de la firma es maximizar sus beneficios sujeta a su restricción presupuestaria, utilizando la ecuación (3) y teniendo en cuenta la restricción presupuestaria siguiente:

$$
M=P_{t}^{H} H_{t}+P_{t}^{K} K_{t}
$$

Donde $H_{t}$ y $K_{t}$ son la fracción de tiempo dedicado al trabajo y el capital privado respectivamente; $P_{t}^{H}$ y $P_{t}^{K}$ son los precios de los factores trabajo y capital, en otras palabras, el salario y el tipo de interés; $M$ es la cantidad de recursos totales disponibles.

Podemos definir la función a maximizar por parte de la firma como:

$$
\max \left[A_{t} K_{t}^{\alpha} H_{t}^{1-\alpha}-\left(M-P_{t}^{H} H_{t}-P_{t}^{K} K_{t}\right)\right]
$$

Sujeto a

$$
\begin{gathered}
K_{t} \geq 0 \\
0 \leq H_{t} \leq 1
\end{gathered}
$$

Resolviendo este problema podemos encontrar las condiciones de primer orden respecto al capital y el trabajo respectivamente:

$$
\begin{array}{r}
p_{t}^{K}=\alpha A_{t} K_{t}^{p(\alpha-1)} H_{t}^{1-\alpha}=r_{t} \\
p_{t}^{H}=(1-\alpha) A_{t} K_{t}^{p \alpha} H_{t}^{-\alpha}=w_{t}
\end{array}
$$




\section{Los hogares}

El hogar representativo maximizará la ecuación (2) sujeto a la siguiente restricción presupuestaria intertemporal:

$$
C_{t} \leq w_{t} H_{t}+r_{t} K_{t}+T_{t}^{f}+K_{t}^{f}-\tilde{p}_{t}^{f} K_{t+1}^{f}-I_{t}
$$

Donde,

$$
\tilde{p}_{t}^{f}=p_{t}^{f}\left[1+\frac{\psi^{*}\left(F D_{t}\right)}{2}\left(\frac{K_{t+1}^{f}-K_{t}^{f}}{K_{t}^{f}}\right)^{2}\right]
$$

Nuevamente, resolviendo para $C_{t}$ y para $H_{t}$, podemos encontrar las ecuaciones de Euler intertemporal para el consumo e intratemporal para el ocio (Obstfeld and Rogoff, 1996, página 115; Kollintzas and Vassilatos, 2000, página 1523), ver desarrollo matemático en el A.2 APÉNDICE 2.

$$
\begin{gathered}
u_{c}\left(C_{t}, 1-H_{t}\right)=\frac{\beta\left(1+r_{t}\right)}{P_{t}^{f}} u_{c}\left(C_{t+1}, 1-H_{t+1}\right) \omega_{t} \\
\mathrm{y} \\
u_{1-H}\left(C_{t}, 1-H_{t}\right)=u_{c}\left(C_{t+1}, 1-H_{t+1}\right) w_{t}
\end{gathered}
$$

Donde, haciendo las modificaciones pertinentes,

$\omega_{t}=\frac{1+P_{t+1}^{f} \psi(.)\left(K_{t+2}^{f} / K_{t+1}^{f}\right)^{2}\left(\left(K_{t+2}^{f}-K_{t+1}^{f}\right) / K_{t+1}^{f}\right)}{1+(\psi(.) / 2)\left(\left(K_{t+1}^{f}-K_{t}^{f}\right) / K_{t}^{f}\right)^{2}+\psi(.)\left(K_{t+1}^{f} / K_{t}^{f}\right)\left(\left(K_{t+1}^{f}-K_{t}^{f}\right) / K_{t}^{f}\right)}(27)$

O alternativamente,

$$
\omega_{t}=\frac{1+P_{t+1}^{f} \psi(.)\left(K_{t+2}^{f} / K_{t+1}^{f}\right)^{2}\left(i_{t+1}^{f}\right)}{1+(\psi(.) / 2)\left(i_{t}^{f}\right)^{2}+\psi(.)\left(K_{t+1}^{f} / K_{t}^{f}\right)\left(i_{t}^{f}\right)}
$$

Donde $i_{t}^{f}$ es la inversión foránea por unidad de capital foráneo, esto es:

$$
i_{t}^{f}=\left(K_{t+1}^{f}-K_{t}^{f}\right) / K_{t}^{f}
$$

Con estas dos condiciones es posible encontrar la senda de evolución del consumo siguiente (ver apéndice A2)

$$
C_{t+1}=(\beta)^{\frac{1}{\sigma}}(1+r)^{1 / \sigma} \omega_{t}^{1 / \sigma}\left(\frac{w_{t}}{w_{t+1}}\right)^{\frac{(1-\gamma)(1-\sigma)}{\sigma}} C_{t}
$$

\section{RESULTADOS Y DISCUSIÓN}

Desde una perspectiva teórica, la metodología de este estudio consiste en comparar los resultados obtenidos en el modelo RBC clásico sin costos de transacción con los obtenidos en este esquema con costos de transacción en el capital foráneo.

La primera diferencia que salta a la vista entre el modelo básico y este esquema está representada en la inclusión de la variable $\psi($.$) en la ecuación (8). Recordemos que en$ un modelo sin costos de transacción la variable $\psi=\psi^{*}=0$, por lo que en la ecuación (11) $\psi()=$.1 y por ende la ecuación (8) toma su forma tradicional. Por tanto, cuanto más grande sea $\psi^{*}$ más costoso será el capital foráneo en el país doméstico, de manera análoga, entre menor sea $\psi^{*}$ menos costoso será el capital foráneo. Esto último sucederá siempre que en la ecuación (13) el desarrollo financiero sea mayor, tal y como se muestra en la Fig. 1

Supongamos por el momento que el costo fijo de transacción es igual a cero $(\bar{\psi}=0)$, en este caso, la ecuación (13) la podemos reescribir como:

$$
\psi^{*}=\frac{1}{F D_{t}}
$$

Si tomamos los límites de $\psi^{*}$ cuando $F D_{t} \rightarrow+\infty$ y cuando $F D_{t} \rightarrow 0^{+}$obtenemos que:

$$
\begin{aligned}
& \lim _{F D_{t} \rightarrow+\infty} \psi^{*}=0 \\
& \lim _{F D_{t} \rightarrow 0^{+}} \psi^{*}=\infty
\end{aligned}
$$

Es decir la primera expresión de la ecuación (31) indica que el costo de transacción tiende a cero cuando el grado de desarrollo 
financiero de los países es muy avanzado, por su parte, la segunda parte indica que, en la medida en que el grado de desarrollo sea menor, los costos de transacción serán muy altos. Si existen costos fijos de transacción $(\bar{\psi} \neq 0)$, el primer límite tenderá a dicho valor y el segundo nuevamente a infinito.

Lo segundo que se debe notar es que, indirectamente, el desarrollo financiero tiene efectos sobre las decisiones óptimas de los individuos, pues en la ecuación (25) se pueden apreciar dos cambios significativos respecto al modelo clásico. El primero de ellos tiene que ver con el factor de descuento utilizado para llevar a valor futuro el consumo presente, en el modelo clásico dicho factor era simplemente $\beta\left(1+r_{t}\right)$, y ahora dicho factor se deflacta por el precio de los activos foráneos, de modo que ahora es $\frac{\beta\left(1+r_{t}\right)}{P_{t}^{f}}$.

El segundo cambio importante se debe a la inclusión del término $\omega_{t}$ al final de dicha ecuación. Es precisamente este término el que permite observar cómo el desarrollo financiero a través de los costos de transacción afecta las decisiones óptimas de los agentes. En la ecuaciones (27) y (28), dados $K_{t+i}^{f}$ y $P_{t+i}^{f} \forall i=0,1,2$, se cumple que:

$$
\frac{\partial \omega_{t}}{\partial \psi(.)}=\frac{4 P_{t+1}^{f}\left(\frac{K_{t+2}^{f}}{K_{t+1}^{f}}\right)^{2} i_{t+1}^{f}-2 i_{t}^{f}\left(i_{t}^{f}+2 \frac{K_{t+1}^{f}}{K_{t}^{f}}\right)}{\left[\left(i_{t}^{f}\right)^{2} \psi(.)+2 i_{t}^{f} \psi(.)+2\right]^{2}}
$$

Con $i_{t}^{f}$ definido según la ecuación (29).

Si $i_{t+i}^{f}<0$ para $i=0,1$ entonces $\frac{\partial \omega_{t}}{\partial \psi(.)}<0$, pero si $i_{t+i}^{f}>0$ el signo es ambiguo, pues depende de la diferencia entre los dos términos del numerador.

$\mathrm{Si}$,

$$
4 P_{t+1}^{f}\left(\frac{K_{t+2}^{f}}{K_{t+1}^{f}}\right)^{2} i_{t+1}^{f}>2 i_{t}^{f}\left(i_{t}^{f}+2 \frac{K_{t+1}^{f}}{K_{t}^{f}}\right) \rightarrow \frac{\partial \omega_{t}}{\partial \psi(.)}>0
$$

Y si,

$4 P_{t+1}^{f}\left(\frac{K_{t+2}^{f}}{K_{t+1}^{f}}\right)^{2} i_{t+1}^{f}<2 i_{t}^{f}\left(i_{t}^{f}+2 \frac{K_{t+1}^{f}}{K_{t}^{f}}\right) \rightarrow \frac{\partial \omega_{t}}{\partial \psi(.)}<0$
Para los casos donde $\frac{\partial \omega_{t}}{\partial \psi(.)}<0, \omega_{t}$ es una función decreciente del costo de transacción $\psi($.$) , o dicho de otra manera, la función \omega_{t}$ es creciente con el desarrollo financiero. Recuerde que la función de costos de transacción $\psi($.$) por construcción es$ decreciente con el nivel de desarrollo financiero, por tanto, cuando la función $\psi($.) crece, es porque el desarrollo financiero decrece y viceversa.

Esto significa que los aumentos en el grado de desarrollo financiero de un país provocan que la función $\omega_{t}$ aumente, y por la ecuación (30) aumentos en $\omega_{t}$ provocan aumentos en $C_{t+1}$, lo que inducirá un aumento en el nivel de producto para que se mantenga el equilibrio de mercado, y por las ecuaciones (21) y (22) habrá un aumento en la remuneración de los factores productivos.

Haciendo unas pequeñas transformaciones podemos reescribir las ecuaciones (21) y (22) así:

$$
r_{t}=\frac{\alpha}{K_{t}} Y_{t} ; w_{t}=\frac{\alpha}{H_{t}} Y_{t}
$$

De esta manera, es fácil ver que aumentos del producto inducen una mayor remuneración factorial.

Este último efecto es el que permite ligar las mejoras en el desarrollo financiero con las reducciones en la pobreza. Si abstraemos el tema de la desigualdad del ingreso, un aumento en la remuneración de los factores productivos hará que los hogares cuenten con más recursos para satisfacer sus necesidades y por ende la pobreza debe reducirse.

Es preciso aclarar que este mecanismo se hace operativo solo bajo las restricciones que se mencionan, a saber que la deuda del país doméstico con el resto del mundo tienda a disminuir, lo que se ve reflejado en el hecho que $i_{t+i}^{f}<0$, además que no existan diferencias significativas en la distribución del ingreso, si existen diferencias 
redistributivas muy amplias, de manera que las ganancias en utilidad dado una mayor posibilidad de consumo futuro gracias a los avances en el desarrollo financiero, el efecto del desarrollo sobre la pobreza será menor, o inclusive podría promover más las desigualdades sociales en términos de ingreso.

\section{CONCLUSIONES}

Este artículo utiliza como base teórica el modelo RBC propuesto por Kollintzas y Vassilatos (2000), con el fin de endogeneizar los costos de transacción en una esquema básico RBC, suponiendo que dichos costos son una función decreciente del desarrollo financiero y, de este manera, observar cómo se afectan las conclusiones del modelo ante variaciones en el desarrollo financiero de los países.

Lo primero en resaltar es que diversos autores han analizado la incidencia del desarrollo financiero en los niveles de pobreza de los países, algunos encuentran evidencia a favor de que el desarrollo financiero es una herramienta útil para reducir la pobreza (Nguyen, Bigman, y Van den Berg, 2007 y Beck, Demirguc-Kun y Levine, 2004), mientras que otros encuentran que no todo desarrollo financiero permite lograr una mejoría en las condiciones de pobreza (Medhi, Ratan, y Toyama, 2009).

Los resultados del modelo indican que mejorías en el desarrollo financiero afectan las decisiones óptimas de los individuos e incide indirectamente en la remuneración de los factores de producción, entre ellos el trabajo.

Con base en los resultados obtenidos, un mayor desarrollo financiero estimulará el consumo futuro, lo que a su vez aumentará la producción, y de esta manera acrecentará la remuneración a los factores; dicho icremento permitirá a los hogares tener más recursos para satisfacer sus necesidades futuras y de esta menra reducir la pobreza de los períodos futuros. Todo este mecanismo descansa sobre los supuestos de que la deuda del país doméstico con el resto del mundo tienda a disminuir, y que no existan diferencias significativas en la distribución del ingreso.

\section{REFERENCIAS}

Beck, T., Demirguc-Kunt, A., \& Levine, R. (2004, December 1). Finance, inequality and poverty: cross-country evidence. 46.

Blanchard, O. (1985). Debt, deficits, and finite horizons. Journal of Political Economy, 93, 223-247.

Bruno, C., \& Portier, F. (1995). A small open economy RBC model: The French Economy Case. In P. Henin, Advances in Business Cycle Research With Aplplication to the Federal and US Economies (pp. 173-194). Berlin: Springer.

Cardia, E. (1991). The dynamics of a small open ecomomy in respond to monetary, fiscal and productivity shocks. Journal of Monetary Economics, 28, 411-434.

Gill, W. (2006, November). The reasons for the low market penetration of banking services in South Africa. 71. Pretoria, South Africa: University of Pretoria.

Kollintzas, T., \& Vassilatos, V. (2000). A small open economy model with transaction costs in foreign capital. European Economic Review, 44, 1515-1541. 
Medhi, I., Ratan, A., \& Toyama, K. (2009). Mobile-Banking Adoptation and Usage by Low-Literate, Low-Income Users int he Developing World. (S.-V. B. Heidelberg, Ed.) Internacionalization, Design, 485494.

Mendoza, E. (1991). Real business cycles in a small open economy. American Economic Review, 81, 797-840.

Nguyen, C., Bigman, D., \& Van den Berg, M. (2007, August 23). Impact of micro credit on poverty and inequality: The Case of The Vietnam Bank for Social Policies. MPRA Munich Personal RePEc Archive, 21.
World Bank. (2013). Remarkable Declines in Global Poverty, But Major Challenges Remain. Retrieved Abril 7, 2014, from

http://www.worldbank.org/en/news /pressrelease/2013/04/17/remarkabledeclines-in-global-poverty-butmajor-challenges-remain

Yaari, M. (1965). Uncertain lifetime, life insurance, and the theory of the consumer. Review or Economic Studies, 32, 137-150. 


\section{A.1 APÉNDICE 1}

Sobre la endogeneización de los costos de transacción a través de la ecuación (13).

De la ecuación (11) se tiene que:

$$
\psi\left(K_{t}^{f}, K_{t+1}^{f}\right)=1+\frac{\psi}{2}\left(\frac{K_{t+1}^{f}-K_{t}^{f} \gamma_{n} \gamma_{z}}{K_{t}^{f}}\right)^{2}
$$

Reemplazando la ecuación (13) en la anterior expresión obtenemos lo siguiente:

$$
\psi\left(K_{t}^{f}, K_{t+1}^{f}\right)=1+\frac{F D_{t} \bar{\psi}+1}{2 F D_{t}}\left(\frac{K_{t+1}^{f}-K_{t}^{f} \gamma_{n} \gamma_{z}}{K_{t}^{f}}\right)^{2}
$$

Tomando primera y segunda derivada respecto a $F D_{t}$ en esta ecuación obtenemos lo siguiente:

$$
\begin{gathered}
\frac{\delta \psi(.)}{\delta F D_{t}}=-\frac{1}{2 F D_{t}{ }^{2}}\left(\frac{K_{t+1}^{f}-K_{t}^{f} \gamma_{n} \gamma_{z}}{K_{t}^{f}}\right)^{2}<0 \\
\frac{\delta^{2} \psi(.)}{\delta F D_{t}{ }^{2}}=\frac{1}{F D_{t}}\left(\frac{K_{t+1}^{f}-K_{t}^{f} \gamma_{n} \gamma_{z}}{K_{t}^{f}}\right)^{2}>0
\end{gathered}
$$

Esto quiere decir que, un aumento en el desarrollo financiero de los países hará que los costos de transacción del capital foráneo se reduzcan, pero que con cada innovación en el sistema financiero que mejora su nivel de desarrollo, dichos costos se reduzcan en menor medida, indicando que cada vez es más difícil reducir los costos con nuevos desarrollos. O leído desde otra perspectiva, cada desarrollo financiero es más difícil de lograr.

\section{A.2 APÉNDICE 2}

El problema de los hogares es maximizar la función de utilidad intertemporal siguiente:

$$
U_{t}=\sum_{s=t}^{\infty} \beta^{s-t} u\left(C_{s}, 1-H_{s}\right)
$$

Sujeto a la restricción intertemporal:

$$
\sum_{s=t}^{\infty}\left(\frac{1}{1+r}\right)^{s-t} C_{s}=(1+r) Q_{t}+\sum_{s=t}^{\infty}\left(\frac{1}{1+r}\right)^{s-t}\left(Y_{s}-I_{s}\right)
$$

Tomando la primera derivada con respecto al consumo se obtiene la ecuación de Euler (25), la cual suponiendo una función de utilidad como la descrita en la ecuación (2) se puede reescribir así:

$\left[C_{t}^{\gamma}\left(1-H_{t}\right)^{1-\gamma}\right]^{-\sigma} C_{t}^{\gamma-1}\left(1-H_{t}\right)^{1-\gamma}=(1-r) \beta\left[C_{t-1}^{\gamma}\left(1-H_{t-1}\right)^{1-\gamma}\right]^{-\sigma} C_{t-1}^{\gamma-1}\left(1-H_{t-1}\right)^{1-\gamma} \omega_{t}$

A su vez, tomando primera derivada con respecto al ocio se obtiene la ecuación (26), la cual suponiendo la misma función de utilidad y organizando términos se obtiene la siguiente ecuación de Euler intratemporal para el ocio:

$$
1-H_{t}=\frac{1-\gamma}{\gamma w_{t}} C_{t}
$$

Ahora, reemplazando esta condición en la ecuación de Euler para el ocio se puede mostrar que la senda que describe la evolución intertemporal del consumo es la siguiente:

$$
C_{t+1}=(\beta)^{\frac{1}{\sigma}}(1+r)^{1 / \sigma} \omega_{t}^{1 / \sigma}\left(\frac{w_{t}}{w_{t+1}}\right)^{\frac{(1-\gamma)(1-\sigma)}{\sigma}} C_{t}
$$

